\title{
Indentation : techniques expérimentales et modélisation multiéchelle
}

\section{Indentation: Experimental techniques and multiscale modelling}

\author{
Anne Habraken ${ }^{1}$, Anne Mertens ${ }^{1}$, Véronique Vitry ${ }^{2}$, Marie-Stéphane Colla ${ }^{3}$, et Eric Le Bourhis ${ }^{4}{ }^{*}$ \\ ${ }^{1}$ Université de Liège, Faculté de Sciences Appliquées, Liège, Belgique \\ ${ }^{2}$ Université de Mons, Faculté Polytechnique, Mons, Belgique \\ ${ }^{3}$ Université de catholique de Louvain, Pôle IMAP, Institut iMMC, Louvain, Belgique \\ ${ }^{4}$ Université de Poitiers, Institut P', UPR 3346, CNRS, Poitiers, France
}

Reçu le 10 janvier 2019 / Accepté le 22 janvier 2019

Du 11 au 14 septembre 2018, à l'université de Liège (Belgique), s'est tenu le colloque Indentation 2018, sixième édition de ce congrès francophone organisé tous les deux ans. Le colloque a réuni tous les scientifiques utilisateurs avancés des techniques d'indentation instrumentée. Ces techniques d'indentation statique ou dynamique sont utilisées largement par l'industrie pour caractériser les surfaces de matériaux fonctionnalisés. Ces techniques récentes ne sont pas encore au stade des essais conventionnels de caractérisation des matériaux massifs. C'était justement l'objet du colloque Indentation 2018: faire un état des lieux des aspects expérimentaux et des modélisations en cours dans le domaine.

Durant ce colloque, qui a réuni 110 participants issus des équipes françaises et européennes possédant des équipements instrumentaux et/ou des moyens numériques dédiés aux techniques d'indentation, environ une quarantaine de présentations orales et une vingtaine de posters ont été présentés. L'ensemble de ces travaux présentés lors de 8 sessions a suscité de nombreuses discussions et échanges entre les participants. Pour la deuxième fois, une formation sur l'indentation a été organisée la veille du colloque (http://www.aimontefiore. org/INDENTATION2018/index. php/program/trainings). Fort du succès rencontré, cette formation sera reconduite lors de la prochaine édition à Lorient en France.

\footnotetext{
* e-mail: Eric.le.bourhis@univ-poitiers.fr
}

Chaque édition du colloque a donné lieu à un numéro spécial dans la revue Matériaux et Technique [1-6]. Inscrit dans cette tradition, nous présentons dans ce numéro thématique, un ensemble d'articles issus d'une sélection de contributions au colloque, publiés après un processus d'évaluation et de révision. Ces articles offrent un aperçu de toutes les thématiques abordées au cours du colloque. L'ensemble du numéro regroupe des travaux fondamentaux et appliqués et permet de faire un point sur des sujets d'actualité dans le domaine de l'indentation.

Les éditeurs (A. Habraken, A. Mertens, V. Vitry, M. S. Colla et E. Le Bourhis) tiennent à remercier les membres du comité d'organisation (L. Duchene, A.M. Habraken, A. Mertens, D. Ruffoni, J.T. Tchuindjang, L. Libralesso, D. Mercier, M.S. Colla, A. Favache, T. Pardoen, S. Ryelandt, L. Bonin, M. Delville, C. Dizier) pour la tenue de ce colloque, ainsi que les membres du comité scientifique du groupe indentation multiéchelle (E. Barthel, S. Benayoun, D. Chicot, J.-P. Guin, V. Keryvin, E. Le Bourhis, J.-L. Loubet, G. Mauvoisin, H. Pelletier, https://sf2m.fr/commissionsthematiques/commission-indentation/) qui ont accepté de rapporter chacun sur plusieurs articles publiés dans cet ouvrage.

\section{Références}

1. S. Benayoun, E. Le Bourhis, Surfaces et interfaces Indentation, rayage et abrasion, Matériaux \& Techniques 93, 185 (2005)

2. O. Bartier, G. Mauvoisin, Indentation : Analyses, développements et applications, Matériaux \& Techniques 96, 9 (2008) 
3. E. Le Bourhis, G. Mauvoisin, Indentation à différentes échelles, Matériaux \& Techniques 99, 161 (2011)

4. S. Bec, S. Benayoun, Indentation: Fondements, développements théoriques et expérimentaux, Matériaux \& Techniques 101, 301 (2013)
5. H. Pelletier, E. Le Bourhis, Comportements mécaniques sous indentation, Matériaux \& Techniques 103, 601 (2015)

6. A. Montagne, D. Chicot, A. Iost, E. Le Bourhis, Indentation : Fondamentaux et développements, Matériaux \& Techniques 105, 101 (2017)

Citation de l'article : Anne Habraken, Anne Mertens, Véronique Vitry, Marie-Stéphane Colla, Eric Le Bourhis, Indentation: techniques expérimentales et modélisation multiéchelle, Matériaux \& Techniques 107, 204 (2019) 